\title{
Evaluation and management of short stature in children
}

\author{
Y A A Jayasena ${ }^{1}$, K Dharshini ${ }^{2}$, K S H de Silva ${ }^{3}$ \\ Sri Lanka Journal of Diabetes Endocrinology and Metabolism 2011; 1: 30-33
}

Poor growth leading to short stature causes enormous psychological consequences and social disadvantages to the child and family, irrespective of the cause. Short stature is defined as height more than 2 standard deviations below the population mean (1) and it is a variant of normal growth pattern in most instances; nevertheless a serious organic pathology needs to be excluded. In spite of advancement of preventive care, still malnutrition is the leading cause of growth retardation in the developing world $(2,3)$.

There is no substitute to a complete clinical history and physical examination in evaluation of a child who is abnormally short. Apart from accurate serial height measurements, growth velocity plays an important role in discriminating from a serious underlying organic cause (4). Further laboratory investigations should only be based on the findings of clinical evaluation.

\section{Clinical evaluation}

\section{History}

A thorough history starting from prenatal period including consanguinity and family history, child's growth and development, i.e. when parents were first concerned about poor growth, questions to assess the significance of the short stature (e.g. shortest in the class, shorter than the younger siblings) and nutritional evaluation should be obtained $(3,5)$ (Table 1$)$.

Table 1

History Importance

Prenatal period

Intra uterine infections, placental insufficiency (PIH, GDM), maternal nutrition / anemia

Neonatal period

Prematurity. Hypoglycemia and neonatal jaundice to suggest pituitary insufficiency

Family history

Consanguinity, heights of parents and siblings and age of puberty

Nutrional history

24 hour dietary recall, quality and quantity of nutrient intake

Review of systems

Exclusion of an intra cranial lesion - headache, vomiting, visual changes. Assessment of cardiac, renal, gastro intestinal systems

Social history and

Psychological impact on the child and family, stressors in home/ school-bullying

psychological

evaluation

PIH - Pregnancy Induced Hypertension, GDM - Gestational Diabetes

\section{Measurement of growth and physical examination (4-6)}

- Accurate height measurement using a standard method, ideally by the same evaluator plotted serially in a growth chart is the key to diagnosis of disorders of growth. The preferred tool for older children would be a standard stadiometer while an infantometer is used for children younger than 3 years with the child being properly positioned.

\footnotetext{
${ }^{1}$ Senior Registrar in Paediatric Endocrinology, Lady Ridgeway Hospital, Colombo, ${ }^{2}$ Senior Registrar in Endocrinology, National Hospital, Sri Lanka, ${ }^{3}$ Cosultant Paediatrician, Lady Ridgeway Hospital, Colombo and Senior Lecturer, Faculty of Medicine, Colombo.
} 
- Upper to lower segment ratio should be evaluated. The average ratio is 1.7 at birth and decreases to 1 by 10 years due to growth of limbs. Presence of skeletal disproportion indicates a skeletal cause for short stature such as achondroplasia. A radiological skeletal survey is required to make an accurate diagnosis.

- Accurate weight measurement along with calculation of body mass index, weight for height, weight for age and height for age is equally important to assess nutritional status.

- Child's adult height potential can be assessed by calculating mid parental height using following formula. This value is plotted in the growth chart corresponding to 20 years.

\section{- Boys: [father's height in $\mathrm{cm}+($ mother's height in $\mathrm{cm}+13 \mathrm{~cm})] / 2$}

- Girls: [(father's height in cm - $13 \mathrm{~cm})+$ mother's height in $\mathrm{cm}] / 2$

- Any clinical features including dysmorphism suggestive of any genetic syndrome should be assessed. E.g. SilverRussel syndrome, Turner syndrome, Noonan syndrome and syndromes associated with obesity such as Prader-Willi syndrome and Down syndrome.

- Physical findings suggestive of important endocrine and systemic causes of short stature and laboratory evaluation of those disorders are illustrated in Table 2.

- Determination of bone age is an important investigation to differentiate endocrine and metabolic causes which manifest as delayed bone age and those that reflect an intrinsic abnormality of the bone where bone age is same as chronological age.

- Any girl with short stature irrespective of the presence or absence of characteristic phenotypic features should have a karyotype to exclude Turner syndrome.

Table 2

\begin{tabular}{lll}
\hline Cause & Clinical features & Evaluation \\
\hline $\begin{array}{l}\text { Hypopituitarism/ } \\
\text { GHDeficiency }\end{array}$ & $\begin{array}{l}\text { Micropenis, midfacial hypoplasia, } \\
\text { midline defects }\end{array}$ & $\begin{array}{l}\text { IGF-I, GH stimulation test, serum cortisol, } \\
\text { thyroid functions }\end{array}$ \\
Cushing syndrome & $\begin{array}{l}\text { Obesity, moon facies, violaceous striae, and } \\
\text { cessation of linear growth }\end{array}$ & $\begin{array}{l}\text { Midnight serum cortisol, 24-hour } \\
\text { urinary free cortisol, dexamethasone } \\
\text { suppression test }\end{array}$ \\
Hypothyroidism & $\begin{array}{l}\text { Increased BMI, sallow complexion, and } \\
\text { delayed relaxation of the deep tendon reflexes }\end{array}$ & $\begin{array}{l}\text { Free thyroxine, thyroid stimulating } \\
\text { hormone (TSH) }\end{array}$ \\
Chronic renal failure & Pallor, hypertension and oedema & Urine analysis, creatinine, Hb, \\
electrolytes, bicarbonate
\end{tabular}

IGF-1 - Insulin like Growth Factor-1, GH - Growth Hormone, PTH - Parathyroid Hormone, ALP - Alkaline Phosphatase BMI - Body Mass Index, FBC - Full Blood Count, Hb - Haemoglobin 


\section{Management}

Management options for a child with short stature would depend on the underlying cause. Short stature secondary to any systemic illness will not reverse unless the underlying cause is evaluated and treated accordingly.

Since malnutrition is a major cause of short stature in developing countries, optimization of nutritional status with appropriate dietary advice or nutritional therapy should be included in the management.

Diagnosis of diseases with genetic implications such as achondroplasia, Prader-Willi syndrome and Laron syndrome necessitates the need for genetic counseling (4).

Treatment of endocrine abnormalities includes replacement of the deficient hormone, i.e. thyroxin in hypothyroidism and growth hormone $(\mathrm{GH})$ therapy for GH deficiency. However, many short children will belong to the group where no active treatment is possible and long term management includes follow up with serial height measurement, height velocity and repeated clinical assessment as necessary.

\section{Growth hormone therapy}

$20 \%$ of children with GH deficiency have an organic cause such as central nervous system tumours, radiation, infection, and traumatic brain injury. Remaining $80 \%$ belong to idiopathic GH deficiency (7). GH therapy should be initiated as soon as the diagnosis is made. However, as GH therapy is expensive, careful judgment of the treatment using cost-benefit analysis and side effects associated with therapy should be taken into consideration before initiation of therapy.

GH therapy is indicated in several other conditions apart from isolated growth hormone deficiency and panhypopituitarism. Turner syndrome, Prader-Willi syndrome, chronic kidney disease, inflammatory bowel disease, persistent postnatal short stature following intrauterine growth retardation and idiopathic short stature (ISS) are some of the other indications for GH therapy (7).

ISS is defined by a height 2.25 or more standard deviations below the mean for age and gender without evidence of underlying disease or GHDeficiency (8). Use of $\mathrm{GH}$ for the ISS indication remains controversial at present (8).

\section{Dose calculation and titration}

$\mathrm{GH}$ is administered daily, in the evening via subcutaneous route. $\mathrm{GH}$ is routinely used in the range of $4.5-9.5 \mathrm{mg} / \mathrm{m}^{2} /$ day. Dosage is periodically adjusted by weight and height and the growth response. Under special circumstances such as Turner syndrome higher doses are required.

\section{Monitoring of therapy and side effects}

Follow up of patients should be conducted on a 3-6 month basis for anthropometry, pubertal development and adverse effects (Benign intracranial hypertension, scoliosis, slipped capital femoral epiphysis) (8). Determination of growth response to $\mathrm{GH}$ is by assessment of increase in height and change in height velocity (1). Bone age should be obtained periodically to reassess height prediction and IGF-1 levels may be helpful in GH dose adjustments (8). Patients with proven or suspected multiple pituitary hormone deficiency are managed similarly and correct recognition, treatment and monitoring of additional hormonal deficiencies $\left(\mathrm{T}_{4}\right.$, cortisol, sex steroids, anti diuretic hormone) is important (1).

\section{Duration of therapy and cessation of therapy}

This is decided by the patient satisfaction with the result of therapy, cost-benefit analysis and side effects. Treatment is stopped when near adult height is achieved, i.e. height velocity $<2 \mathrm{~cm} /$ year and/or bone age $>16$ year in boys and $>14$ year in girls (8).

\section{Counseling}

Many short children are very conscious of being different, and in some cases this will lead to considerable psychological impact on the child leading to interference with academic performance and personality development. In order to prevent this psychological impact the following options may be attempted and this is very important where no active treatment is possible (4).

1. Informed explanation of the exact cause and prognosis of the condition.

2. Objective reassurance: Child should be seen, three to six monthly and he or she should be shown the progress in growth and puberty.

3. Redirection of activities: Morale of the child can be maintained by directing him or her to access to activities suitable for the stature or to activities where height is unimportant such as non sport activities or indoor games.

Nevertheless the treatment and follow up should focus on managing the whole child and not merely the short stature and continued counseling and psychological support should be available to the child and family.

\section{References}

1. Consensus Guidelines for the Diagnosis and Treatment of Growth Hormone (GH) Deficiency in Childhood and Adolescence: Summary Statement of the GH Research Society. The Journal of Clinical Endocrinology and Metabolism 2000; 85(11): 3990-3.

2. World Health Organization Department of Nutrition for Health and Development. WHO Global Database on Child Growth and Malnutrition. Available at: http://www.who.int/ nutrition/databases/en/. Accessed July 28, 2011. 
3. Martorell R. The nature of child malnutrition and its longterm implications. Food and Nutrition Bulletin 1999; 20(3): 288-92.

4. Warne G L. The assessment of growth in children. Australian Family Physician 1982; 11(6): 422-7.

5. Nwosu BU, Lee MM. Evaluation of short and tall stature in children. American Family Physician 2008; 78(5): 597-604.

6. Halac I, Zimmerman D. Evaluating short stature in children. Pediatric Annals 2004; 33(3): 170-6.
7. Hardin DS. Treatment of short stature and growth hormone deficiency in children with somatotropin (rDNA origin). Biologics: Targets and Therapy 2008; 2(4): 655-61.

8. Cohen $\mathrm{P}$ et al. Consensus Statement on the Diagnosis and Treatment of Children with Idiopathic Short Stature: A Summary of the Growth Hormone Research Society, the Lawson Wilkins Pediatric Endocrine Society, and the European Society for Paediatric Endocrinology Workshop. The Journal of Clinical Endocrinology and Metabolism 2008; 93(11): 4210-17. 